\title{
ITU-R Standardization Activities on Cognitive Radio Systems
}

\author{
Stanislav Filin ${ }^{1}$, Homare Murakami ${ }^{1}$, Hiroshi Harada ${ }^{1}$, Hitoshi Yoshino ${ }^{2}$, Kanshiro Kashiki ${ }^{3}$, Tatsuo Shibata ${ }^{4}$ \\ ${ }^{1}$ NICT, ${ }^{2}$ Softbank Mobile, ${ }^{3}$ KDDI R\&D Labs, ${ }^{4}$ ATR \\ Japan \\ sfilin@nict.go.jp, homa@nict.go.jp, harada@nict.go.jp, \\ hitoshi.yoshino@mb.softbank.co.jp,ka-kashiki@kddilabs.jp,shibata@atr.jp
}

\begin{abstract}
The current radio environment is characterized by its heterogeneity. Different aspects of this heterogeneity include: multiple operators and services, various radio access technologies, different network topologies, broad range of radio equipment, and multiple frequency bands. Such environment has a lot of technical and business opportunities. The examples are: joint management of several radio access networks within one operator to balance load of these networks; detecting and using unused spectrum in the allocated frequency bands without interrupting the operation of the primary users of such frequency bands; spectrum trading between several operators. To exploit such opportunities, the concept of Cognitive Radio System (CRS) has been developed. A lot of CRS usage scenarios and business cases are possible. This has triggered a lot of standardization activity at all levels, including ITU, IEEE, ETSI, and ECMA, where each of these organizations considers multiple CRS deployment scenarios and business directions. This paper presents current ITU-R standardization activities on CRS. ${ }^{1}$
\end{abstract}

Keywords-component; ITU; cognitive radio system; standardization

\section{INTRODUCTION}

The current radio environment is characterized by its heterogeneity. Different aspects of this heterogeneity include: multiple operators and services, various radio access technologies, different network topologies, broad range of radio equipment, and multiple frequency bands.

Such environment has a lot of technical and business opportunities. The examples are: joint management of several radio access networks within one operator to balance load of these networks; detecting and using unused spectrum in the allocated frequency bands without interrupting the operation of the primary users of such frequency bands; spectrum trading between several operators.

To exploit such opportunities, the concept of Cognitive Radio System (CRS) has been developed. A lot of CRS usage scenarios and business cases are possible. This has triggered a lot of standardization activity on all levels, including ITU,

${ }^{1}$ This research was conducted under a contract of R\&D for radio resource enhancement, organized by the Ministry of Internal Affairs and Communications, Japan.
IEEE, and ETSI, where each of this organizations consider multiple CRS deployment scenarios and business directions.

This paper presents current ITU-R activities on CRS.

Before WRC-07, a common concern within ITU-R was the protection of existing services from potential interference from SDR and CRS systems. WRC-07 adopted new agenda item 1.19 requiring WRC-12 to consider regulatory measures for the introduction of SDR and CRS.

WRC-07 adopted Resolution 956 (WRC-07), inviting ITU$\mathrm{R}$ to study the need for regulatory measures related to the application of SDR and CRS. The resolution also resolved that WRC-12 should consider the results of the studies and take the appropriate actions. The task of conducting the necessary studies was assigned to ITU-R WP 1B. Also, ITU-R WP 5A studies technical aspects of Cognitive Radio Systems.

The rest of this paper is organized as follows. Section 2 introduces WRC-12 agenda item 1.19. Sections 3 and 4 describe ITU-R WP1B and WP5A activities on CRS. Section 5 concludes this paper.

\section{WRC-12 AGENDA ITEM 1.19 ON CRS}

WRC-07 adopted new agenda item 1.19 requiring WRC-12 to consider regulatory measures for the introduction of SDR and CRS. The agenda item 1.19 is "to consider regulatory measures and their relevance, in order to enable the introduction of software-defined radio and cognitive radio systems, based on the results of ITU R studies, in accordance with Resolution 956 (WRC 07)."

In this regard, WRC-07 adopted Resolution 956 (WRC-07) on "Regulatory measures and their relevance to enable the introduction of software-defined radio and cognitive radio systems" [1].

The rationale for this resolution includes:

- Cognitive radio and self-configuring networks are expected to provide additional flexibility and improved efficiency to the overall spectrum use

- ITU-R is already studying such advanced radio technologies, their functionalities, the key technical characteristics, requirements, performance and benefits 
- Considerable research and development is being carried out on cognitive radio systems and related network configurations such as self-configuring networks

- Cognitive radio systems may cover a number of radio access techniques (RATs)

- Cognitive radio systems include self-configuring networks of different network topologies that will be able to set their spectrum usage based on the locally available spectrum

- Some studies indicate usefulness to have means to assist in the determination of the local spectrum usage, such as wireless or wired access to a database or to other networks

- Some studies indicate a possible need for a worldwide harmonized cognitive supporting pilot channel with a bandwidth less than $50 \mathrm{kHz}$, whilst other studies indicate that the availability of a database could support access and connectivity, and therefore support the use of these systems.

The resolution resolves to invite ITU-R to study whether there is a need for regulatory measures related to the application of CRS technologies and whether there is a need for regulatory measures related to the application of SDR. It further resolves that WRC-12 consider the results of these studies and take the appropriate actions.

The task of conducting the necessary studies was assigned to ITU-R WP $1 \mathrm{~B}$.

\section{ITU-R WP1B ACTIVITIES ON CRS}

Within agenda item 1.19, ITU-R WP1B has developed two documents related to CRS:

- Definitions of SDR and CRS [2]

- Draft CPM text on WRC-12 agenda item 1.19 [3].

\section{A. Definitions of SDR and CRS}

Under the framework of WRC-12 agenda item 1.19, ITU-R WP1B has developed definitions of SDR and CRS to assist in the conduct of studies, to make clear the distinctions between SDR and CRS technologies, and to provide common understanding and facilitate their use in an unambiguous way in ongoing work by the ITU-R. Below are these two definitions.

"Software-defined radio (SDR): A radio transmitter and/or receiver employing a technology that allows the RF operating parameters including, but not limited to, frequency range, modulation type, or output power to be set or altered by software, excluding changes to operating parameters which occur during the normal pre-installed and predetermined operation of a radio according to a system specification or standard."

“Cognitive radio system (CRS): A radio system employing technology that allows the system to obtain knowledge of its operational and geographical environment, established policies and its internal state; to dynamically and autonomously adjust its operational parameters and protocols according to its obtained knowledge in order to achieve predefined objectives; and to learn from the results obtained."

\section{B. Draft CPM text on WRC-12 agenda item 1.19}

With regard to $\mathrm{CRS}$, the following key elements can be identified in the draft CPM text.

1) CRS deployment scenarios

ITU-R WP1B has identified four deployment scenarios:

1. Use of CRS technology to guide reconfiguration of connections between terminals and multiple radio systems

2. Use of CRS technology by an operator of radiocommunication systems to improve the management of its assigned spectrum resources

3. Use of CRS technology as an enabler of cooperative spectrum access

4. Use of CRS technology as an enabler of opportunistic spectrum access.

In the first scenario, multiple radio systems employing different radio access technologies are deployed on different frequencies to provide wireless access. Two possible examples of this scenario are identified.

In one example, some terminals are reconfigurable and can adjust their operational parameters and protocols to use different radio access technologies.

In another example, some terminals have the capability to communicate with different radio systems, e.g. based on the subscriptions, but they cannot reconfigure their operational parameters and protocols to use different radio access technologies. Additional stations can be deployed to serve as a bridge between multiple radio systems and terminals.

To illustrate the second scenario, consider an operator who already owns a network and operates in assigned spectrum and decides to deploy another network, based on a new generation radio interface technology in the same or other assigned spectrum, covering the same geographical area. Taking into consideration the non-uniform nature of radiocommunication needs within this area, an operator having more than one network based on different radio technologies could dynamically and jointly manage the deployed resources, in order to adapt the configuration of the networks to maximize the overall network capacity.

In the third scenario, information on spectrum use is exchanged amongst the radio systems in order to avoid mutual interference. Two examples are identified for such cooperative spectrum access.

In the first example, the radio systems take advantage of parts of the unused spectrum resulting from variations in the occupancy of the assigned spectrum in a specific location at a specific time.

In the second example, CRS technology is used for managing interference between private and public radio systems using the same frequency band. 
In the fourth scenario, CRS may access parts of unused spectrum in bands shared with other radio systems without causing harmful interference.

\section{2) CRS challenges and opportunities}

A common concern within the ITU-R is the protection of existing services from potential interference from the services implementing CRS technology, especially from the dynamic spectrum access capability of CRS.

In addition, a service using CRS should not adversely affect other services in the same band with the same or higher status. Thus, the introduction and operation of stations using CRS technologies in systems of any radiocommunication service should not impose any additional constraints to other services sharing the band.

3) CRS capabilities and their applicability to facilitate coexistence in shared bands

The ITU-R has identified a set of capabilities of CRS that may facilitate coexistence with existing systems. The following elements could be considered as examples of capabilities of CRS:

- $\quad$ spectrum sensing

- $\quad$ positioning (geo-location)

- access to information on the spectrum usage, local regulatory requirements and policies

- capabilities to adjust operational parameters.

4) Relationship between SDR and CRS

$\mathrm{SDR}$ is recognized as an enabling technology for the CRS. SDR does not require characteristics of CRS for operation. Either technology can be deployed/implemented without the other.

In addition, SDR and CRS are at different phases of development, i.e., radiocommunication systems using applications of SDR have been already utilized and CRS are now being researched and applications are under study and trial.

\section{5) Analysis of the results of studies}

The potential benefits and applicability of CRS technologies to various radiocommunication services is recognized as well as the fact that CRS would be introduced in some services.

There would be a need for further studies on CRS technology, addressing especially dynamic and/or opportunistic spectrum access.

The use of CRS in some bands used by particular radiocommunication services may require the development of unique ITU-R Recommendations and Reports to address these issues. However, the study concluded that there is no need for modification to the Radio Regulations for this Agenda item for the introduction of CRS technology.

6) Methods to satisfy the agenda item

Two methods are proposed to satisfy the agenda item:

1. No change to the Radio Regulations
2. Add a WRC Resolution providing guidance for further studies and guidance for the use of CRS and no other changes to the Radio Regulations.

The first method allows administration to facilitate implementation of CRS. However, it does not provide guidance for the studies and provisions for the implementation of CRS.

The second method allows administrations to facilitate implementation of CRS. Also, the resolution provides a framework for guidance on further studies. However it does not provide provisions for the implementation of CRS. In another option of the second method the resolution provides guidance to administrations for the use of CRS.

\section{ITU-R WP5A ACTIVITIES ON CRS}

Within agenda item 1.19, ITU-R WP5A is developing report on "Cognitive radio systems in the land mobile service" [4]. This report focuses on technical aspects of CRS. The work is still ongoing. The following key elements can be identified in the draft report text.

\section{A. Technical Characteristics and Capabilities}

The following key capabilities of the CRS are underlined in its definition:

- Capability to obtain knowledge

- Capability to adjust operational parameters and protocols

- Capability to learn.

Capability to obtain knowledge is one of three key characteristics of CRS. Such knowledge includes the following key components:

- CRS operational radio environment

- CRS operational geographical environment

- Internal state of CRS

- $\quad$ Established policies

- Usage patterns

- Users' needs.

The CRS operational radio environment is characterized, for example, by current status of spectrum usage, indication of available radio systems and their assigned frequency bands, coverage areas of these radio systems, interference levels.

The CRS operational geographical environment is characterized, for example, by positions of radios which are components of CRS and other radio systems, by orientation of antennas of radios of CRS and other radio systems, by distribution of users in the geographic area of CRS.

The internal state of CRS can be characterized by configuration of CRS (e.g., frequency bands and protocols used by its radios), traffic load distribution, transmission power values.

The established policies may describe frequency bands allowed to be used by CRS under certain conditions, where 
such conditions may include maximum level of transmission power in operating and adjacent frequency bands, rules that CRS shall follow to avoid causing harmful interference.

The usage patterns may collect behavior of CRS, other radio systems, and users.

The users' needs may be described by user preferences or policies. Examples of such user preferences are request for high bandwidth, low delay, fast download time, and low cost.

In order to obtain knowledge, CRS can use various approaches, including:

- Collecting information from component radio systems

- Geo-location

- $\quad$ Spectrum sensing

- White space data base access

- Access to Cognitive Pilot Channel.

Component radio systems of CRS typically perform a lot of measurements, such as RSSI, SINR, load, etc. Also, they are aware of their current state, for example, frequency bands and RATs used by base stations and terminals, transmission power values, etc. All this information contributes a lot to the knowledge of CRS.

Positions of radios (e.g., base stations and terminals) that are components of CRS and other radio systems can be obtained using geo-location. Geo-location can be performed during professional installation or using a localization system, e.g., GPS and wireless positioning system.

White space database access and spectrum sensing is very important in some deployment scenarios of CRS. These two approaches are used to detect primary users and to identify white spaces and may be also used to detect secondary users.

Access to Cognitive Pilot Channel (CPC) is also very important in some deployment scenarios of CRS. The CPC serves as a means to exchange knowledge between components of the CRS and in such cases CPC is typically considered as a part of the CRS.

The second key characteristic of the CRS is its capability to dynamically and autonomously adjust its operational parameters and protocols according to the obtained knowledge in order to achieve some predefined objectives. Such adjustment consists of two stages: making decision and reconfiguration.

Typically, the CRS includes intelligent management system responsible for making decisions on which parameters and protocols need to be adjusted, when, and how. Following the decisions made, CRS performs reconfiguration of its radios. Such reconfiguration may include change of one or several of the following parameters:

- Output Power

- Frequency band

- $\quad$ Radio access technology.
These three examples of the parameters that may be adjusted by the CRS are regulated parameters. This means that they are typically specified by radio regulations. This is the new capability of the CRS, compared to commonly used power control or adaptive modulation and coding.

The third key characteristic of the CRS is its capability to learn from the results of its actions in order to further improve its performance.

Figure 1 summarizes general architecture of the CRS concept. The main components of the CRS are the intelligent management system and the reconfigurable radios. The four main actions of the CRS are obtaining knowledge, making decisions, reconfiguration, and learning.

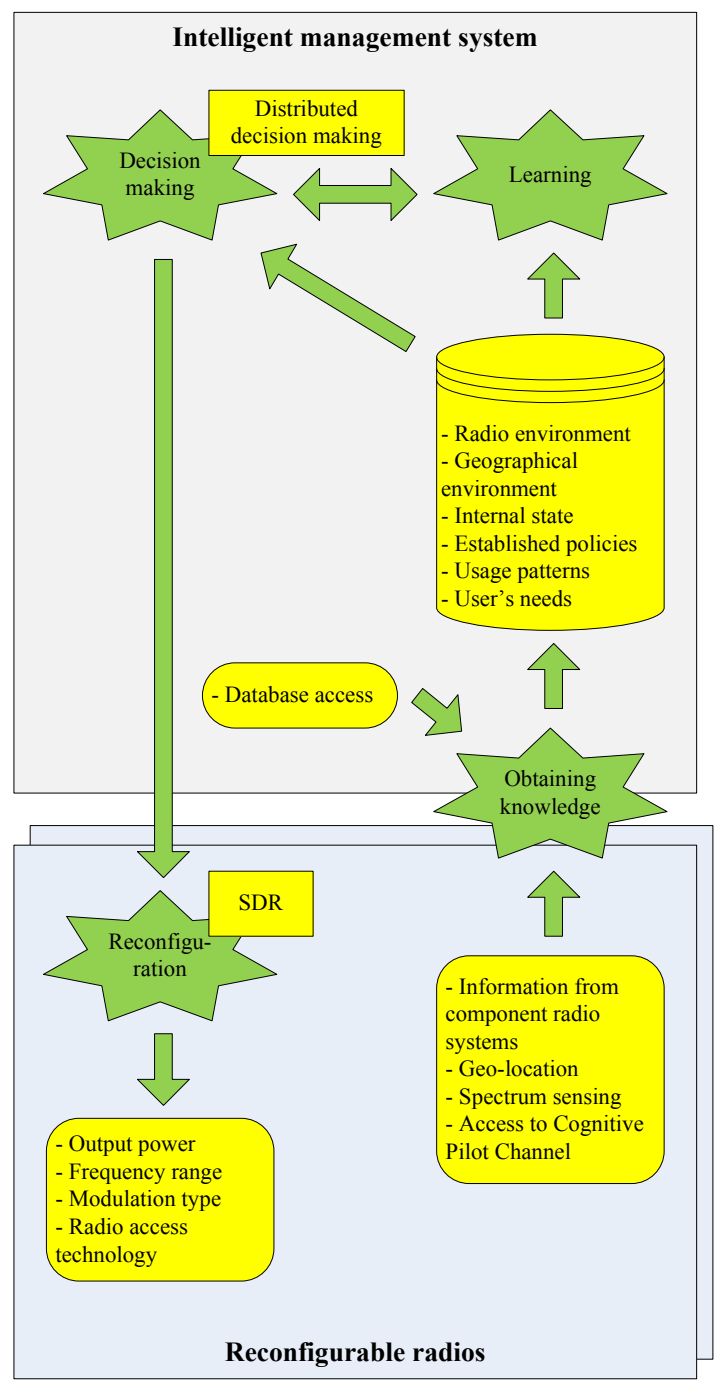

Figure 1. General architecture of the CRS concept.

\section{B. Operational techniques}

Many technologies have contributed to the CRS concept. Among them are the following key technologies:

- Distributed decision making

- Cognitive Pilot Channel

- White space access 
- Whitespace database access and spectrum sensing

- $\quad$ Software Defined Radio.

The distributed decision making refers to the architecture of the management system inside the CRS responsible for making decisions on which parameters and protocols need to be adjusted, when, and how. Also, such management system will control the process of obtaining knowledge.

Depending on the deployment scenario, the CRS can include a lot of radio nodes distributed on a large territory. It is very difficult to manage such large CRS by one centralized management entity. More efficient and scalable approach is to develop distributed architecture of the CRS management system, where management functions are distributed between network side and terminal side, as well as, inside network.

For example, to perform network-terminal distributed decision making, the policy based management approach can be used. If terminal reconfiguration is considered, reconfiguration policies can be generated inside network and sent to terminals. Then, terminals can make final reconfiguration decisions within framework defined by these reconfiguration policies.

The concept of the Cognitive Pilot Channel (CPC) assumes that the CRS management system has distributed architecture. Given such assumptions, there is a need for communication link between network side and terminal side of the management system. The CPC provides such link.

In general, two types of the CPC are distinguished: in-band $\mathrm{CPC}$ and out-band CPC. In the in-band CPC, the management system uses the same communication link that is used for user data transmission. The out-band CPC uses a separate wireless system dedicated for exchanging management information between network and terminals of the CRS.

The white space access refers to the spectrum usage scenario where the radio regulations allow the CRS to access temporary unused spectrum in the frequency bands allocated to another radio system. When such spectrum usage scenario is considered, the radio system to which frequency bands are allocated is called "primary system," while the CRS is called "secondary system." The temporary unused spectrum is called "white space."

In white space access scenario, the CRS accesses spectrum on a secondary basis. Correspondingly, the CRS shall not cause harmful interference to the primary system. This means that the CRS is allowed to transmit in a particular part of spectrum only if there is no primary system operation in this spectrum. When the primary system starts operation in a particular part of its spectrum, the CRS shall stop transmission in this spectrum.

Two methods are currently identified for primary system protection: white space database access and spectrum sensing. White space database is the database that contains information on primary systems operation in a particular frequency bands or channels in particular locations. The CRS is allowed to start operation only if the white space database indicates that there is no primary system operation in some frequency bands in locations where the CRS is deployed. The CRS is only allowed to use frequency bands indicated by the white space database.

The white space database information about the primary systems starting operation in their frequency bands has some delay. This delay is equal to the delay between the time when a primary system starts transmission and the time when such information is reflected in the white space database and distributed to the CRS. During such delay, the white space database cannot be used to protect the primary system starting transmission from the harmful interference caused by the CRS operation. To minimize such harmful interference, the CRS shall perform spectrum sensing in its operational frequency bands in order to detect the primary system starting operation. If the primary system is detected, the CRS shall stop operation.

The capability to adjust operational parameters is one of the key capabilities of the CRS. In many scenarios, the radio nodes of the CRS change their operating frequency bands and RATs. While it is not mandatory, but the most convenient way to reconfigure radio nodes is by using Software Defined Radio (SDR) technology.

\section{CONCLUSIONS}

Currently a lot of standardization activity related to Cognitive Radio Systems is performed on all levels, including ITU, IEEE, and ETSI, where each of this organizations consider multiple CRS deployment scenarios and business directions. This paper has presented current ITU-R standardization activities on Cognitive Radio Systems.

Before WRC-07, a common concern within ITU-R was the protection of existing services from potential interference from SDR and CRS systems. WRC-07 adopted new agenda item 1.19 requiring WRC-12 to consider regulatory measures for the introduction of SDR and CRS.

WRC-07 adopted Resolution 956 (WRC-07), inviting ITU$\mathrm{R}$ to study the need for regulatory measures related to the application of SDR and CRS. The resolution also resolved that WRC-12 should consider the results of the studies and take the appropriate actions. The task of conducting the necessary studies was assigned to ITU-R WP 1B. Also, ITU-R WP 5A studies technical aspects of Cognitive Radio Systems.

This paper has introduced WRC-12 agenda item 1.19, and ITU-R WP1B and WP5A standardization activities on Cognitive Radio Systems.

\section{REFERENCES}

[1] Resolution 956 (WRC-07), "Regulatory measures and their relevance to enable the introduction of software-defined radio and cognitive radio systems," 2007.

[2] Report ITU-R SM.2152, "Definitions of Software Defined Radio (SDR) and Cognitive Radio System (CRS)," Sep. 2009.

[3] Annex 5 to WP1B Chairman's Report 1B/267-E, "Draft CPM Text on WRC-12 Agenda Item 1.19," Jul. 2010.

[4] Annex 12 to WP5A Chairman's Report 5A/601-E, "Working Document towards a Preliminary Draft New Report,” Nov. 2010. 